\title{
Postnatal Maturation of Phosphodiesterase 5 (PDE5) in Piglet Pulmonary Arteries: Activity, Expression, Effects of PDE5 Inhibitors, and Role of the Nitric Oxide/Cyclic GMP Pathway
}

\author{
LAURA MORENO, BEGOÑA LOSADA, ANGEL L. COGOLLUDO, FEDERICA LODI, \\ CLAIRE LUGNIER, EDUARDO VILLAMOR, MANUEL MORO, JUAN TAMARGO, AND \\ FRANCISCO PÉREZ-VIZCAÍNO
}

Department of Pharmacology (L.M., A.L.C., F.L., J.T., F.P.-V.), Department of Pediatrics (B.L., M.M.), School of Medicine, University Complutense of Madrid, 28040 Madrid, Spain, Laboratoire de Pharmacologie et Physico-Chimie (C.L.), CNRS-UMR 7034, 67401 Illkirch, France, and Department of Pediatrics (E.V.), University Hospital Maastricht, Research Institute Growth and Development, Maastricht 6202 AZ, The Netherlands

\begin{abstract}
ABST
After birth and during the first days of extrauterine life,
pulmonary arterial pressure is progressively reduced to reach the
adult values. We hypothesized that changes in PDE5 activity
might be involved in the pulmonary postnatal maturation of the
nitric oxide (NO)/cGMP pathway. The PDE5 inhibitor sildenafil
produced vasorelaxant responses in isolated pulmonary arteries.
These effects were similar in newborn (3-18 h) and 2-wk-old
piglets, unchanged by endothelium removal, and markedly in-
hibited by the soluble guanylyl cyclase inhibitor ODQ. The peak
of the transient vasorelaxant response to NO gas increased with
postnatal age but was unaffected by PDE inhibition. However,
the duration of the response to NO was significantly increased.
The vasorelaxant response to sodium nitroprusside was potenti-
ated by sildenafil in both age groups. The PDE5 inhibitors
dipyridamole and zaprinast, produced qualitatively similar ef-
fects but with lower potency. Both total and PDE5-dependent
\end{abstract}
Successful adaptation of the newborn to postnatal conditions requires a dramatic transition of the pulmonary circulation. During the first minutes of extrauterine life, as the lung becomes responsible for blood oxygenation and there is a marked reduction in pulmonary vascular resistance $(1,2)$. A second phase of pulmonary vascular resistance reduction takes place over the first $2 \mathrm{wk}$ of extrauterine life in pigs and humans to reach the pulmonary pressure values characteristic of the adult

Received September 26, 2003; accepted April 16, 2004.

Correspondence: Francisco Pérez-Vizcaíno, Ph.D., Department of Pharmacology, School of Medicine, University Complutense, E-28040 Madrid, Spain; email: fperez@med.ucm.es

Supported by grants from CICYT (SAF 2002/02304) and CAM (08.4/0036.2001). A. C. and L.M. were supported by FIS and by Ministerio de Educación Cultura y Deporte, respectively.

DOI: 10.1203/01.PDR.0000139412.58594.D0
cGMP hydrolytic activity and PDE5 protein expression increased with postnatal age. All these results suggest that PDE5 is a key regulator of NO-induced vasodilation in the postnatal pulmonary arteries. PDE5 inhibition is able to produce pulmonary vasodilation even in the absence of a functional endothelium and potentiates the vasorelaxant response to exogenous $\mathrm{NO}$ and nitroprusside. However, PDE5 is not responsible for the maturational increase of NO bioactivity during the first days of extrauterine life. (Pediatr Res 56: 563-570, 2004)
Abbreviations
ANP, atrial natriuretic peptide
NO, nitric oxide
PDE, cyclic nucleotide phosphodiesterase (EC 3.1.4.17)
PPHN, persistent pulmonary hypertension of the newborn

$(1,3)$. Several groups have consistently reported a low activity of the NO/cGMP pathway for smooth muscle vasodilation during the first hours of extrauterine life in rabbit, lamb, and piglet pulmonary arteries $(4-8)$. This NO activity increases during the first $2 \mathrm{wk}$ in parallel with the postnatal reduction in pulmonary arterial pressure. Some infants fail to achieve or sustain the normal decrease in pulmonary vascular resistance at birth, which leads to severe respiratory distress and hypoxemia, referred to as PPHN $(1,9)$.

cGMP is a key second messenger in the regulation of vascular smooth muscle tone (10) whose intracellular concentrations are tightly controlled by the balance between its synthesis and hydrolysis. cGMP is synthesized by a family of guanylyl cyclases (10), whose activities are mainly controlled by NO and ANP. cGMP is hydrolyzed by cyclic nucleotide 
PDE, a superfamily of isozymes consisting of at least 11 families, PDE1 to PDE11 $(11,12)$. Thus, the vasodilator/ antiproliferative effects of NO and ANP in the pulmonary circulation can be limited by the activity of PDE. PDE5 is a cGMP-specific PDE isoform whereas PDE1 can hydrolyze both cAMP and cGMP, and both isoforms are abundant in the lung (11-14). PDE5 activity is increased in pulmonary arteries from rats with chronic hypoxia-induced pulmonary hypertension (14) and in lambs with pulmonary hypertension after in utero aortopulmonary vascular graft placement (15) or with PPHN induced by compression of the ductus arteriosus in utero (16). Moreover, the specific PDE5 inhibitor sildenafil has been recently reported to be a selective pulmonary vasodilator useful for the treatment of adult pulmonary hypertension (17-19). In addition, sildenafil lowers pulmonary vascular resistance in an experimental model of PPHN (20) and anecdotal use of sildenafil in PPHN have also been reported (21). Maturational changes in PDE5 activity and expression have been reported in mouse, rat, and sheep whole lung homogenates and proposed to be involved in the postnatal decrease in pulmonary vascular resistance (22-24). However, in the lung, PDE5 is present, at least, in pulmonary arteries, pulmonary veins, trachea, bronchi, and epithelium (25-28), and little is known about the maturation of PDE5 specifically in pulmonary arteries and its functional role in the regulation of the NO/cGMP pathway in the pulmonary circulation during early life.

Therefore, we hypothesized that PDE activity could modulate vascular tone and the vasorelaxant responses to the NO/cGMP pathway in neonatal pulmonary arteries and that changes in PDE5 activity and PDE5 protein expression might be involved in the postnatal maturation of the NO/cGMP pathway.

\section{METHODS}

All the procedures conform with the Guide for the Care and Use of Laboratory Animals (National Institutes of Health publication No. 85-23, revised 1996) and were approved by our institutional review board.

Tissue preparation. Male piglets of 3-18 h (newborn, $n=$ 32) or 15-20 d of age ( $2 \mathrm{wk}, n=40)$ from a local farm were used in this study. The lungs were rapidly immersed in cold $\left(4^{\circ} \mathrm{C}\right)$ Krebs solution (composition in $\mathrm{mM}: \mathrm{NaCl} 118, \mathrm{KCl}$ 4.75, $\mathrm{NaHCO}_{3} 25, \mathrm{MgSO}_{4} 1.2, \mathrm{CaCl}_{2} 2.0, \mathrm{KH}_{2} \mathrm{PO}_{4} 1.2$, and glucose 11). The pulmonary arteries (third branch with an internal diameter of about $0.5-1.5 \mathrm{~mm}$ ) were carefully dissected free of surrounding tissue and cut into rings of $2-3 \mathrm{~mm}$ length $(8,29)$. Except where otherwise stated, the endothelium was removed by gently rubbing the intimal surface of the rings with a metal rod. The endothelium removal procedure was verified by the inability of acetylcholine $\left(10^{-6} \mathrm{M}\right)$ to relax arteries precontracted with noradrenaline $\left(10^{-6} \mathrm{M}\right)$.

Contractile tension recording. Rings were mounted between two hooks in a 5-mL organ bath filled with Krebs solution at $37^{\circ} \mathrm{C}$ gassed with a $95 \% \mathrm{O}_{2} / 5 \% \mathrm{CO}_{2}$ gas mixture. At this concentration, $\mathrm{O}_{2}$ did not modify the relaxant response to NO in previous studies (7). The arteries were stretched to their optimal resting tension ( 0.5 and $0.7 \mathrm{~g}$ for newborn and 2-wk animals, respectively), at which the rings produced the maximal contractile response to $\mathrm{KCl}$ in previous experiments. The contraction was measured by an isometric force transducer using data acquisition software and hardware as previously described $(8,29)$. Rings were precontracted with the thromboxane $\mathrm{A}_{2}$ mimetic 9,11-dideoxy-11 $\alpha, 9 \alpha$-epoxymethanoprostaglandin $\mathrm{F}_{2 \alpha}\left(\mathrm{U} 46619,10^{-7} \mathrm{M}\right)$ which induced a submaximal contractile response $(430 \pm 31 \mathrm{mg}, n=32$ and $1012 \pm 76$ $\mathrm{mg}, n=40$, in newborn and 2-wk-old piglets, respectively). In previous experiments, these contractions were equally effective in the two age groups when expressed as a percentage of the responses induced by $40 \mathrm{mM} \mathrm{KCl} \mathrm{(8).} \mathrm{Then,} \mathrm{concentration-}$ response curves to the PDE inhibitors, sodium nitroprusside, and forskolin were carried out by cumulative addition of the drugs. As sildenafil was the most potent and selective PDE5 inhibitor, this drug was preferentially used for further experiments. Due to the rapid disappearance of NO gas in the bath, the curves to NO were performed in a noncumulative fashion by addition of increasing volumes of Krebs solution saturated with NO. In some experiments, U46619-stimulated rings were treated for $20 \mathrm{~min}$ with the soluble guanylyl cyclase inhibitor ODQ $(3 \mu \mathrm{M})$, the nonselective inhibitor of NO synthase (NOS) $\mathrm{N}$-nitro-L-arginine methyl ester (L-NAME) $(0.1 \mathrm{mM})$ or the selective inhibitor of the inducible form of NOS (iNOS) $1400 \mathrm{~W}(10 \mu \mathrm{M})$. The concentration-response curves to NO or nitroprusside were also performed in arteries pretreated for 20 $\mathrm{min}$ in the presence of PDE inhibitors at concentrations producing threshold vasorelaxant responses $(10-30 \%)$. To prepare the NO saturated solutions, a $20-\mathrm{mL}$ vial of Krebs solution was initially bubbled with $\mathrm{N}_{2}$ for $15 \mathrm{~min}$ and then continuously bubbled with NO (450 ppm) as described (29). The concentration of $\mathrm{NO}$ in the saturated solution was measured by an amperometric NO-sensitive electrode (ISO-NO, WPI, Sarasota, FL). The vehicle DMSO at the maximal concentration used $(0.1 \%)$ had no significant effect on U46619-induced tone or NO-induced vasorelaxation.

Western blot analysis. Pulmonary arteries were isolated, frozen in liquid nitrogen, and stored at $-80^{\circ} \mathrm{C}$. Piglet mesenteric arteries of a similar diameter and piglet platelets were used as positive controls. Platelets were obtained by centrifuging citrated (3.8\%) blood at $120 \mathrm{~g}$ for $10 \mathrm{~min}$, the supernatant was further centrifuged at $1000 \mathrm{~g}$ for $10 \mathrm{~min}$ and the pellet stored at $-80^{\circ} \mathrm{C}$. Frozen arteries and platelets were homogenized in a glass potter in $300 \mu \mathrm{L}$ of a buffer of the following composition: $10 \mathrm{mM}$ HEPES (pH 8), $10 \mathrm{mM} \mathrm{KCl}, 1 \mathrm{mM}$ EDTA, $1 \mathrm{mM}$ EGTA, $1 \mathrm{mM}$ DTT, $40 \mu \mathrm{g} / \mathrm{mL}$ aprotinin, 4 $\mu \mathrm{g} / \mathrm{mL}$ leupeptin, $4 \mu \mathrm{g} / \mathrm{mL} N \alpha$-p-tosyl-L-lysine chloromethyl ketone, $5 \mathrm{mM} \mathrm{NaF}, 10 \mathrm{mM} \mathrm{Na}_{2} \mathrm{MoO}_{4}, 1 \mathrm{mM} \mathrm{NaVO}_{4}$, and 0.5 $\mathrm{mM}$ phenylmethanesulfonyl fluoride. The homogenate was centrifuged at 100,000 $\mathrm{g}$ for $30 \mathrm{~min}$. The supernatant (cytosolic fraction) was collected and the protein content was determined using the Bradford assay (reagents from Bio-Rad, Hercules, CA). Western blotting was performed with $20 \mu \mathrm{g}$ of protein from the cytosolic fraction of pulmonary or mesenteric arteries, $12 \mu \mathrm{g}$ of protein from platelet homogenates, and $2 \mu \mathrm{L}$ of a purified bovine PDE5 standard (Calbiochem, Darmstadt, Germany) per lane. SDS-PAGE (7.5\% acrylamide) was performed using the method of Laemmli in a mini-gel system (Bio-Rad). Samples from newborns and 2-wk-old animals were run in 
parallel. The proteins were transferred to polyvinylidene difluoride membranes overnight, incubated with rabbit anti-PDE5 polyclonal antibody (1:1000, Calbiochem), and then with an anti-rabbit secondary horseradish peroxidase conjugated antibody. The bands were visualized by chemiluminescence (ECL, Amersham Pharmacia Biotech UK, Ltd., Little Chalfont, buckinghamshire, UK) and quantified using an image analysis software (TotalLab, Nonlinear Dynamics, Newcastle upon Tyne, UK). The results were expressed as a percentage of the data of newborn animals.

PDE activity. PDE activity was measured by a two-step radioenzymatic assay, as previously described (30). Assay was performed at a substrate concentration of $1 \mu \mathrm{M}\left[{ }^{3} \mathrm{H}\right] \mathrm{cGMP}$, with or without addition of the PDE5 inhibitor zaprinast (10 $\mu \mathrm{M})$. QAE-Sephadex A 25 columns were used to separate the final products (guanosine) from the residual substrate (cGMP). The specific activity of PDE5 was measured by subtracting the activity in the presence of zaprinast from the total cGMP-PDE activity.

Drugs. U46619 was from Alexis Biochemicals (Läufelfingen, Switzerland), ODQ (1H- [1,2,4]oxadiazolo[4,3-a]quinoxalin-1-one) from Tocris Cookson Ltd (Bristol, UK), sildenafil was a gift from Pfizer (New York, NY) and all other drugs from Sigma Chemical Co. (Alcobendas, Spain). Drugs were dissolved initially in distilled deionized water (except for ODQ, dipyridamole, zaprinast, and vinpocetine in DMSO) to prepare a $0.01 \mathrm{M}$ stock solution and further dilutions were made in Krebs solution.

Statistical analysis. Results are expressed as means \pm SEM and $n$ reflects the number of animals from which the arterial rings were obtained. Individual cumulative concentration-response curves were fitted to a logistic equation. The drug concentration exhibiting 30\% relaxation was calculated from the fitted concentration-response curves for each ring and expressed as negative $\log$ molar $\left(-\log \left[\mathrm{IC}_{30}\right]\right)$. The half-life of the transient vasorelaxant response to $\mathrm{NO}$ was calculated by measuring the time from the peak relaxant response to $50 \%$ recovery. Statistically significant differences between groups were calculated by $t$ test for unpaired observation or for multiple comparisons by an ANOVA followed by a Newman-Keuls test. A value of $p<0.05$ was considered statistically significant.

\section{RESULTS}

Vasorelaxant effects of PDE inhibitors. In endotheliumintact pulmonary arteries from newborn and 2-wk-old piglets stimulated with U46619 $(0.1 \mu \mathrm{M})$, the specific PDE5 inhibitor sildenafil $(0.1 \mathrm{nM}-1 \mu \mathrm{M})$ produced concentration-dependent vasorelaxant responses (Fig. 1). These effects were not significantly different in newborn and in 2-wk-old animals (Table 1). The modulatory role of the endothelium was analyzed by comparing the activity in intact and endothelium-denuded arteries. Endothelium removal had no effects on the vasorelaxation induced by sildenafil (Fig. 1). The role of basal cGMP was analyzed using the inhibitor of soluble guanylyl cyclase ODQ $(3 \mu \mathrm{M})$. Administration of ODQ in pulmonary arteries stimulated with U46619 substantially increased the vasoconstriction $(68 \pm 6.8 \%$ and $68 \pm 12.9 \%$ of the previous tone in newborn
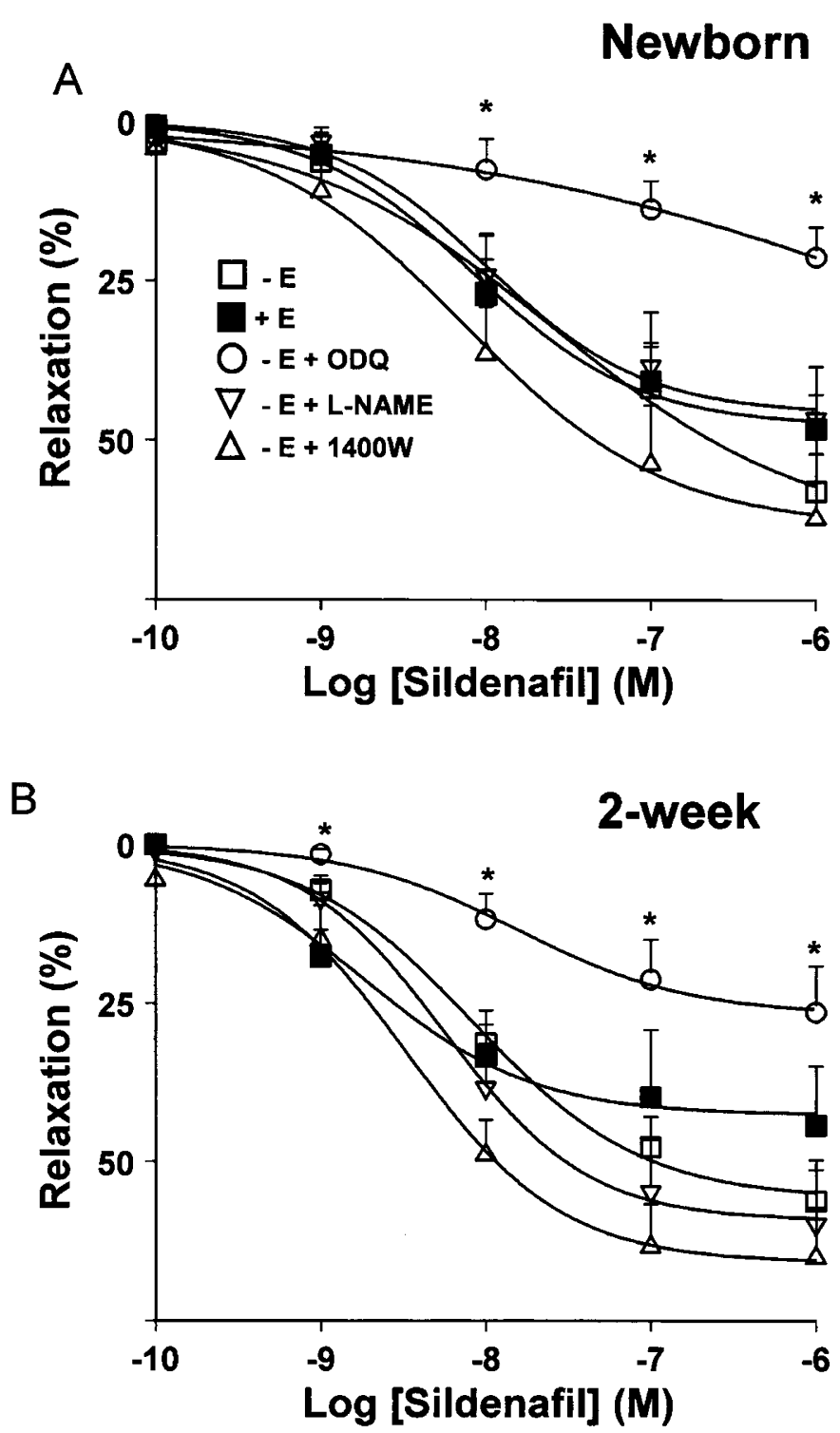

Figure 1. Vasorelaxant effects of sildenafil in intact (+E) or endotheliumdenuded $(-E)$ pulmonary arteries from newborn and 2-wk-old piglets stimulated with $0.1 \mu \mathrm{M}$ U46619. Relaxation induced by sildenafil in denuded arteries was not affected by L-NAME $(0.1 \mathrm{mM})$ or $1400 \mathrm{~W}(10 \mu \mathrm{M})$ but inhibited by ODQ $(3 \mu \mathrm{M})$. Results are expressed as means $\pm \operatorname{SEM}(n=5-17)$. $* p<0.05 v s-\mathrm{E}$.

and 2-wk-old animals, respectively). The role of NO synthesized by NOS in the relaxant effect induced by sildenafil was also studied. Treatment with the nonselective NOS inhibitor L-NAME $(0.1 \mathrm{mM})$ or with the iNOS inhibitor $1400 \mathrm{~W}(10 \mu \mathrm{M})$ had no effect on the relaxation induced by sildenafil (Fig. 1).

The PDE5 inhibitors zaprinast $(0.1-10 \mu \mathrm{M})$ and dipyridamole $(0.1-10 \mu \mathrm{M})$, which inhibit PDE1 at higher concentrations, were 100 - to 300 -fold less potent than sildenafil on vasorelaxation (Table 1), as described on PDE5 activity $(31,32)$, and their effects were similar in both age groups. The effects of zaprinast were also unaffected by endothelium removal but inhibited by ODQ (not shown). The PDE1 inhibitor vinpocetine produced a clear vasorelaxant response only at high concentrations $(100 \mu \mathrm{M})$ and the effect was also similar in both 
Table 1. Potency ( $p I C_{30}$ values) of PDE inhibitors and NO to induce relaxation in endothelium-denuded arteries (calculated from Figs. 1 and 2)

\begin{tabular}{cccccc}
\hline & Sildenafil & Zaprinast & Dipyridamole & Vinpocetine & NO \\
\hline Newborn & $7.65 \pm 0.39$ & $5.04 \pm 0.15$ & $5.55 \pm 0.28$ & $4.23 \pm 0.17$ & $6.92 \pm 0.16$ \\
2 wk old & $7.92 \pm 0.14$ & $5.29 \pm 0.18$ & $5.78 \pm 0.14$ & $4.31 \pm 0.14$ & $7.72 \pm 0.16^{*}$ \\
\hline
\end{tabular}

Results are expressed as means $\pm \operatorname{SEM}(n=6-18)$.

$* p<0.01$ vs newborn.

age groups (Table 1). However, the effects of vinpocetine were not affected by ODQ (not shown).

Effects of PDE inhibition on NO- and nitroprussideinduced pulmonary vasorelaxation. The possible potentiation of NO gas- and nitroprusside-induced pulmonary vasorelaxation was analyzed in the presence of a PDE inhibitor concentration eliciting a $10-30 \%$ relaxation. The peak relaxant response to $\mathrm{NO}$ gas increased significantly with postnatal age (e.g. $39 \pm 5.2 \%$ and $65.7 \pm 7.9 \%$ of the previous tone at 200 nM NO in newborn and 2-wk-old animals, respectively; $p<$ 0.01 ), as previously described (8). These peak relaxant responses were not affected by sildenafil $(3 \mathrm{nM})$ in either age group (Fig. 2, $A$ and $B$ ). Higher concentrations of sildenafil (10 $\mathrm{nM}), 10 \mu \mathrm{M}$ zaprinast, $1 \mu \mathrm{M}$ dipyridamole, or $30 \mu \mathrm{M}$ vinpocetine were also without significant effect (not shown). However, the analysis of the kinetics of the responses induced by a single concentration of NO $(200 \mathrm{nM})$ revealed that, although the durations of the response were similar in the two age groups (half-lives of $85 \pm 8 \mathrm{~s}$ and $88 \pm 17 \mathrm{~s}$ in newborn and 2 -wk-old animals, respectively), they were significantly increased $(p<0.05)$ by 3 and $10 \mathrm{nM}$ sildenafil, $10 \mu \mathrm{M}$ zaprinast, and $1 \mu \mathrm{M}$ dipyridamole in both age groups (Fig. 2, $C$ and $D$ ). In contrast, the PDE1 inhibitor vinpocetine $(30 \mu \mathrm{M})$ had no effect on the duration of the response to NO (Fig. 2C).

The specific PDE5 inhibitor sildenafil, at $3 \mathrm{nM}$, significantly increased nitroprusside-induced vasorelaxation (Fig. 3) in the older animals (3.4-fold, $p<0.05$ ) but not in newborns (2.2fold, NS), whereas at $10 \mathrm{nM}$ it increased nitroprusside-induced vasorelaxation at both ages (9.6- and 16.8-fold for newborn and 2-wk-old animals, respectively, $p<0.05$ for both). However, sildenafil $(10 \mathrm{nM})$ had no effect on the relaxant responses to the adenylyl cyclase activator forskolin in either age group (not shown). The potency of nitroprusside in pulmonary arteries from newborn animals also increased in the presence of zaprinast $(10 \mu \mathrm{M})$ by 6.2 -fold $(p<0.05)$, whereas dipyridamole $(1 \mu \mathrm{M})$ had no significant effect (1.2-fold increase). In the older animals, the potency of nitroprusside was significantly increased by the two inhibitors (8.7- and 14.7 -fold, respectively). In contrast, the PDE1 inhibitor vinpocetine (30 $\mu \mathrm{M})$, which by itself reduced U46619-induced contractions by $13 \pm$ $3 \%$ and $26 \pm 6 \%$ in newborn and 2 -wk-old piglets, respectively, had no effect on nitroprusside-induced relaxation in either age group (not shown).

Total PDE and specific PDE5 activity. The hydrolysis of cGMP in homogenates of pulmonary arteries was strongly inhibited by the PDE5 inhibitor zaprinast $(10 \mu \mathrm{M})$, so that PDE5 accounted for a $63 \pm 2 \%$ and $66 \pm 2 \%$ of the total PDE activity in newborn and 2-wk-old animals, respectively $(n=$ 4-6). Both total and specific PDE5 cGMP hydrolysis increased with postnatal age (Fig. 4).
PDE5 expression. In Western blots using an antibody directed toward purified bovine PDE5, two bands of approximately 93-98 kD were detected in the cytosolic fraction of piglet pulmonary arteries (Fig. 5A). The heavier band co-migrated with purified bovine PDE5 standard and was also present in piglet platelets and piglet mesenteric arteries, which were used as positive controls, whereas the lighter one was only present in the mesenteric arteries. Preliminary experiments indicated that these bands were almost undetectable in particulate fractions from pulmonary arteries (not shown), which is consistent with the poor PDE5 activity previously found in the particulate fraction of homogenates from bovine and rat pulmonary arteries $(31,32)$. Consistent with the age-dependent increase in PDE5 activity, an increased expression of cytosolic PDE5 was observed in 2-wk-old animals when compared with newborns (Fig. 5B). The lower band observed in the Western blots was similar in the two age groups, and its nature is presently unknown but it might represent a lower molecular weight 5'-splice variant of PDE5 (e.g. PDE5A2) $(13,33)$.

\section{DISCUSSION}

In the present study, we have analyzed the postnatal maturation of PDE5 and its role in the regulation of vascular tone in piglet pulmonary arteries. The results can be summarized as follows: 1) The specific PDE5 inhibitor sildenafil produced a vasorelaxant response that did not require the presence of a functional endothelium but was dependent on the activity of soluble guanylyl cyclase. 2) The potency of sildenafil was similar in newborns and 2-wk-old animals. 3) Sildenafil did not modify the peak but prolonged the duration of the relaxant responses to NO similarly in the two age groups. 4) In contrast, it increased the relaxant potency of sodium nitroprusside at both ages. 5) The PDE5 inhibitors zaprinast and dipyridamole, which also inhibit PDE1 at high concentrations, produced qualitatively similar effects but with a lower potency. 6) PDE5 accounted for $65 \%$ of the total cGMP-PDE activity and both total and PDE5-specific cGMP hydrolysis and PDE5 protein expression increased with postnatal age.

PDE5 is expressed in a variety of organs, including lung, placenta, kidney, aorta, platelets, corpora cavernosa, and several brain regions $(13,33)$. PDE5 activity has been reported to be the major metabolic pathway for cGMP in pulmonary arteries and, therefore, an important contributor in the regulation of pulmonary arterial tone $(31,32)$. In the present study, PDE5 accounted for approximately $65 \%$ of the cyclic-GMP hydrolytic activity in pulmonary arteries from newborns and 2-wk-old piglets, i.e. results similar to those reported in bovine (31) and rat pulmonary arteries (32). This prominent role of PDE5 in pulmonary arterial tone was consistent with the potent 

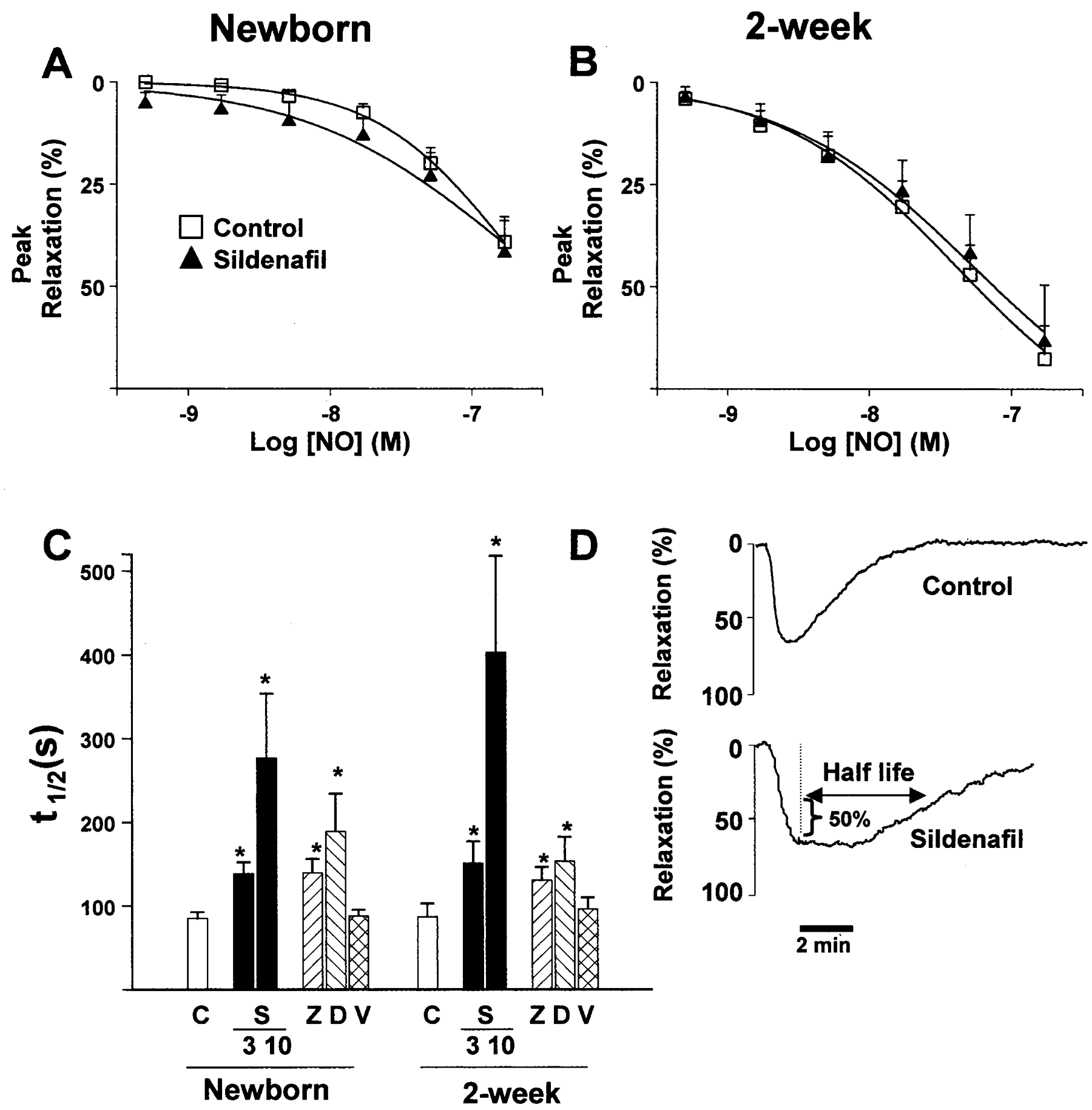

Figure 2. Vasorelaxant effects of NO in endothelium-denuded pulmonary arteries from newborn and 2-wk-old. Panels $A$ and $B$ show the peak relaxant responses to increasing concentrations of NO animals in the absence (control) or presence of sildenafil $(3 \mathrm{nM})$. Panel $C$ shows the half-life of the vasorelaxant effects of NO in control $(C) 3$ or $10 \mathrm{nM}$ sildenafil $(S), 10 \mu \mathrm{M}$ zaprinast $(Z), 1 \mu \mathrm{M}$ dipyridamole $(D)$, or $30 \mu \mathrm{M}$ vinpocetine $(V)$, and panel $D$ a representative recording of the time course of the response to NO in pulmonary arteries from 2-wk-old animals in the absence (control) and in the presence of sildenafil (10 nM). Results are expressed as means $\pm \operatorname{SEM}(n=4-17) .{ }^{*} p<0.05$ vs control.

vasorelaxant effects of the PDE5 inhibitor sildenafil. The fact that vinpocetine, which is supposed to be a specific PDE1 inhibitor (11), required high concentrations to induce vasorelaxant responses that were unaffected by inhibition of soluble guanylyl cyclase and was unable to inhibit bovine vascular and rat cardiac PDE1 $\left(\mathrm{IC}_{50}>100 \mu \mathrm{M}\right.$, personal unpublished data), suggests that it is not appropriate to characterize PDE1 $(34,35)$.
Dipyridamole and zaprinast inhibit PDE5 at low $(<1 \mu \mathrm{M})$ and PDE1 at higher concentrations $(>10 \mu \mathrm{M})(11,32)$. Their vasorelaxant effects appear to be due mainly to their inhibitory effects on PDE5 because, at the concentration used, they potently inhibited PDE5 and they showed a vasorelaxant and NO-potentiating profile similar to sildenafil and different from vinpocetine. 

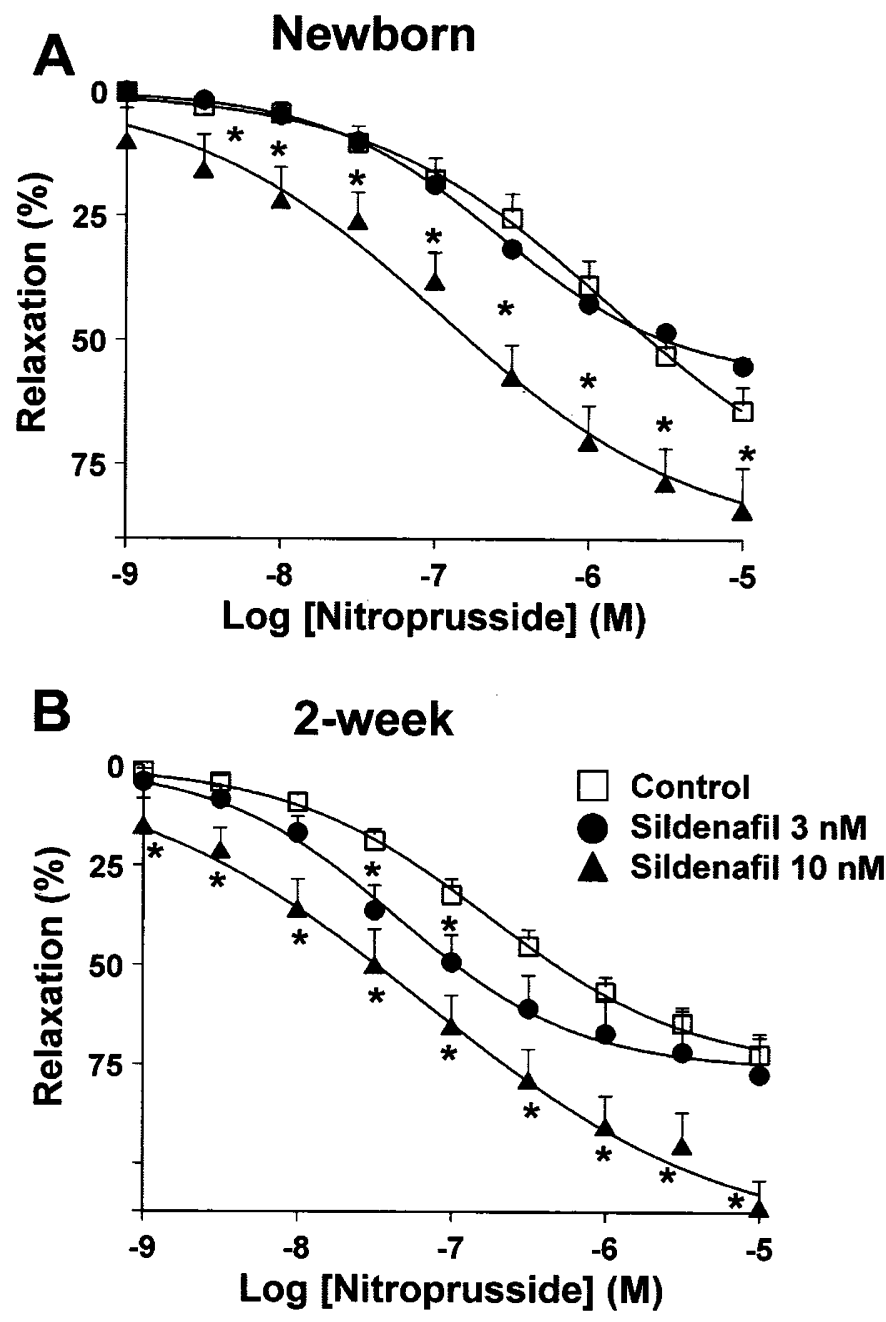

Figure 3. Vasorelaxant effects of sodium nitroprusside in endotheliumdenuded pulmonary arteries from newborn $(A)$ and 2-wk-old $(B)$ animals in the absence (control) or presence of sildenafil $(3 \mathrm{nM}$ and $10 \mathrm{nM})$. Results are expressed as means $\pm \operatorname{SEM}(n=6-15) . * p<0.05 v s$ control.

As previously described in rat pulmonary arteries (33), the vasorelaxant effects of PDE5 inhibitors were unaffected by endothelium removal, suggesting that PDE5 activity is mainly present in the media layer. Furthermore, our experiments indicate that basal release of NO from the endothelium is not required for the vasorelaxant effects of PDE5 inhibitors. However, ODQ strongly reduced the vasorelaxant effects of sildenafil and zaprinast, suggesting that basal activity of soluble guanylyl cyclase produces cGMP concentrations sufficient to induce a strong vasorelaxant effect when PDE5-dependent cGMP hydrolysis is reduced. NO can theoretically be produced by the inducible form of NO synthase (iNOS) in vascular smooth muscle and stimulate guanylyl cyclase. However, this does not seem to be the case in our preparations inasmuch as iNOS inhibition with $1400 \mathrm{~W}$ had no effect on the relaxation induced by sildenafil, indicating that NO synthesized by iNOS is not required for basal cGMP levels. A possible residual effect of eNOS after endothelium removal can be also excluded because the nonselective NOS inhibitor L-NAME had no effect on sildenafil-induced relaxation. As expected, PDE5 inhibitors increased the relaxant responses to agents stimulating soluble

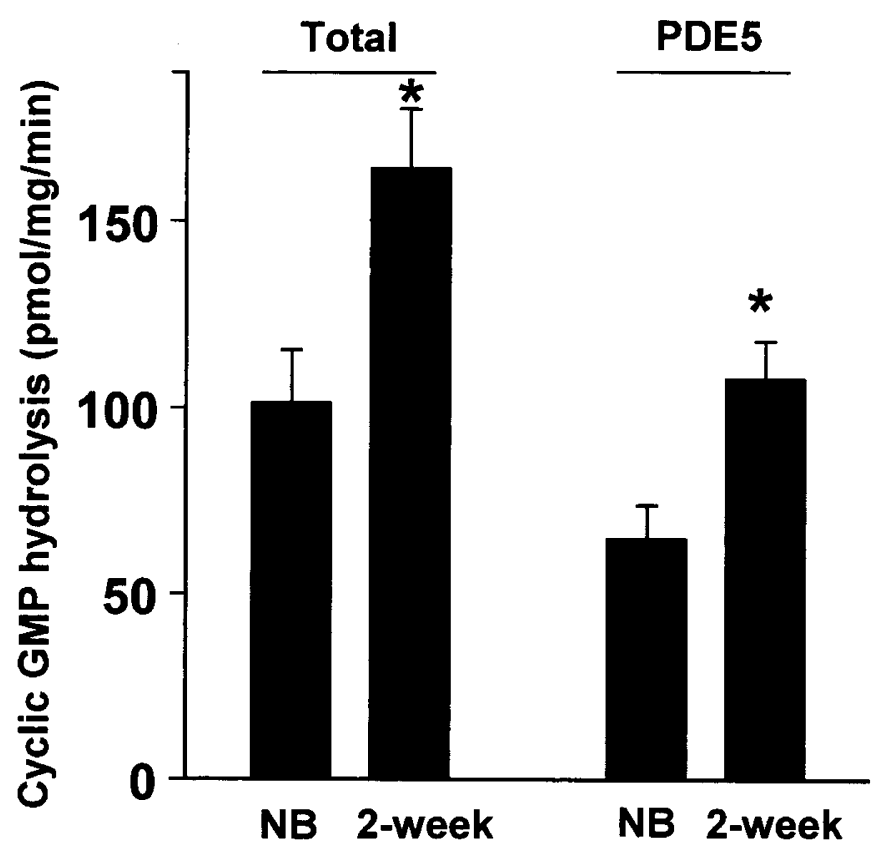

Figure 4. Total and PDE5 cGMP PDE activity in homogenates of pulmonary arteries from newborn $(N B)$ and 2-wk-old piglets. Results are expressed as means $\pm \operatorname{SEM}(n=4-6) .{ }^{*} p<0.05$ vs NB.

\section{A \\ B}
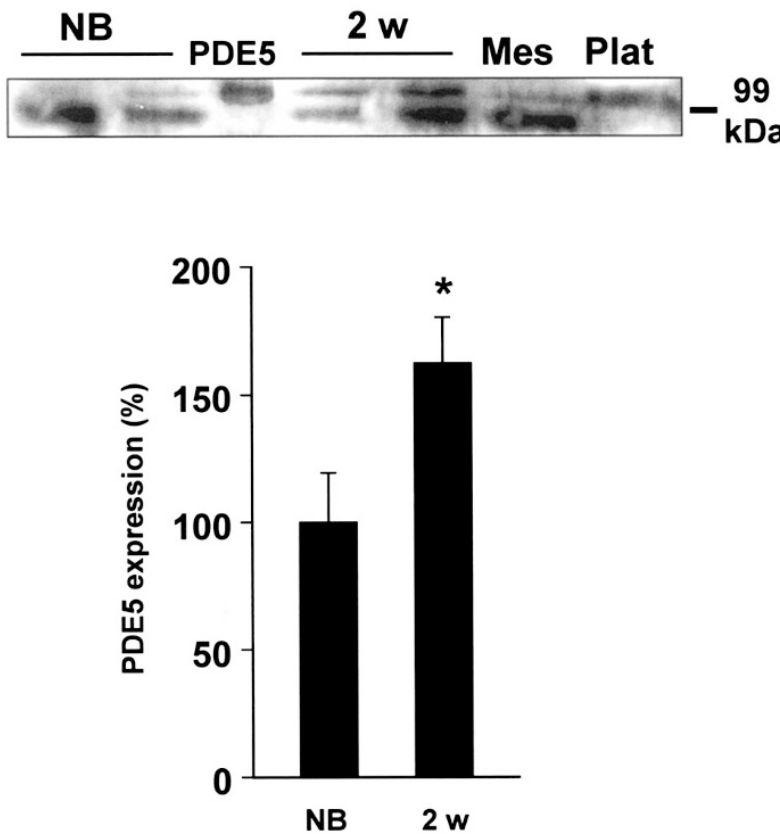

Figure 5. PDE5 expression in the cytosolic fraction of homogenates of pulmonary arteries from newborn $(N B)$ and 2-wk-old animals and of mesenteric arteries (Mes) and platelets (Plat) from 2-wk-old piglets. PDE5 indicates purified bovine PDE5 (panel A). Panel B shows the densitometric analysis of PDE5 expression in pulmonary arteries. The results are expressed as a percentage of the data of newborn animals, means $\pm \operatorname{SEM}(n=4-5) . * p<0.05$ vs NB.

guanylyl cyclase (i.e. NO and nitroprusside). However, there was a different effect of PDE5 inhibitors on NO- as compared with nitroprusside-induced relaxations. The fact that PDE5 inhibitors did not change the peak relaxant response to NO can be explained by the short time of exposure of the arteries to $\mathrm{NO}$ 
and by the rapid and transient nature of the relaxation compared with that of nitroprusside (29). The half-life of NO gas in the tissue bath is approximately $15 \mathrm{~s}(29)$, so that the cGMP that induces the relaxant response is expected to be synthesized in high amounts during a short time and then the rate of decay of intracellular concentrations of cGMP would depend on PDE activity. Thus, the maximum cGMP would be unaffected by PDE5 activity. In this case, PDE5 inhibitors would prolong the duration but not the maximal relaxant response to $\mathrm{NO}$, as we have observed. However, when the stimulation of guanylyl cyclase is more sustained, as occurs with nitroprusside, cGMP concentrations depend on the balance of its synthesis and hydrolysis rates and inhibition of PDE5 is expected to produce a progressive cGMP accumulation. Therefore, in contrast to the result with $\mathrm{NO}$ gas, the concentration-response curves to nitroprusside were shifted to the left by PDE5 inhibitors.

An increase in PDE5 activity and/or expression during the early postnatal period has been found in mouse, rat, and sheep whole lung homogenates (22-24). In the lung, PDE5 is present, at least, in pulmonary arteries, pulmonary veins, trachea, bronchi, and epithelium (25-28). In the present study, we have analyzed the activity and expression of PDE5 specifically in pulmonary arteries from newborn and relatively mature piglets and their functional correlate, i.e. the vasorelaxant and NOpotentiating effects of PDE5 inhibitors. PDE5 activity increased with postnatal age. Even when we found no age-dependent statistical differences in the effects of individual PDE5 inhibitors, there was a trend for an increase with age in the vasorelaxant effects of the three inhibitors tested and also a trend for a stronger potentiation by PDE5 inhibitors of nitroprusside-induced relaxation in the older animals, which are likely to be related to the age-dependent change in PDE5 activity. PDE5 activity has been reported to be modulated by cGMP through a protein kinase G-dependent phosphorylation (36). However, PDE5 expression also increased postnatally suggesting that changes in activity result from increased gene expression rather than changes in the phosphorylative status of the PDE5 protein.

During the first hours of extrauterine life there is a low responsiveness of pulmonary arteries to endothelium-derived or exogenous NO, which increases during the first $2 \mathrm{wk}$ in parallel with the postnatal reduction in pulmonary arterial pressure (4-8). Changes in PDE5 activity would be a potential candidate to explain this maturational process. However, PDE5 activity and expression was lower in newborns, which should lead to increased rather than reduced NO bioactivity. Furthermore, the peak relaxant response to NO increased with postnatal age but was unaffected by PDE5 inhibitors. Inasmuch as this response is almost abolished by ODQ (8), the lack of effect of PDE5 inhibitors cannot be due to a cGMP-independent mechanism. On the contrary, the half-life of the vasorelaxant response to NO, which was sensitive to inhibition of PDE5, did not change postnatally. Taken together, these results indicate that PDE5 is not responsible for the low responsiveness to NO of pulmonary arteries after birth. However, as opposed to NO, the vasorelaxant responses to carbon monoxide (CO) in piglet pulmonary arteries, which are also due to activation of soluble guanylyl cyclase, decreased with postnatal age (7). Moreover, the responses to 8-bromo cGMP, a membrane permeable ana- $\log$ of cGMP, which is relatively resistant to hydrolysis by PDE were not significantly modified by postnatal age (7). Thus, the increase in PDE5 activity in pulmonary arteries is consistent with this decrease in the responsiveness to CO. Therefore, the different maturational profile of the responsiveness to $\mathrm{NO}$ and $\mathrm{CO}$ probably reflects changes in the bioavailability of NO in the smooth muscle cells (8).

Inhaled NO, is an effective pulmonary vasodilator used in near-term or term infants with PPHN. However, it fails to improve oxygenation in about $50 \%$ of the patients and does not change mortality (37), which has led to the search for alternative treatments. Recently, sildenafil has been proposed as a useful therapy for the treatment of adult pulmonary hypertension (17-19,38). In fact, the combination of inhaled NO and sildenafil seems to lower pulmonary vascular resistance in a synergistic manner (19). The prominent role of PDE5 in cGMP hydrolysis and the effective vasorelaxant effects of sildenafil in neonatal pulmonary arteries as described herein together with the effectiveness in experimental models of PPHN (20) supports a role for this drug in human persistent neonatal pulmonary hypertension. However, in a recent report, the pulmonary vasodilation induced by sildenafil in a porcine model of meconium aspiration syndrome was accompanied by a deleterious effect on arterial oxygenation (39).

In conclusion, taken together, these results suggest that PDE5 is a key regulator of NO-induced vasodilation in the postnatal pulmonary arteries. PDE5 inhibition is able to produce pulmonary vasorelaxation even in the absence of a functional endothelium and prolongs and/or increases the vasorelaxant response to exogenously added NO and nitroprusside. However, PDE5 does not seem to be responsible for the maturation of NO bioactivity during the first days of extrauterine life.

\section{REFERENCES}

1. Fineman JR, Soifer SJ, Heymann MA 1995 Regulation of pulmonary vascular tone in the perinatal period. Annu Rev Physiol 57:115-134

2. Ziegler JW, Ivy DD, Kinsella JP, Abman SH 1995 The role of nitric oxide, endothelin, and prostaglandins in the transition of the pulmonary circulation. Clin Perinatol 22:387-403

3. Haworth SG, Hislop AA 1981 Adaptation of the pulmonary circulation to extrauterine life in the pig and its relevance to the human infant. Cardiovasc Res 15:108-119

4. Morecroft I, MacLean MR 1998 Developmental changes in endothelium-dependent vasodilation and the influence of superoxide anions in perinatal rabbit pulmonary arteries. Br J Pharmacol 125:1585-1593

5. Abman SH, Chatefield BA, Rodman DM, Hall SL, McMurtry IF 1991 Maturational changes in endothelium-derived relaxing factor activity of ovine pulmonary arteries in vitro. Am J Physiol 260:L280-L285

6. Shaul PW 1999 Regulation of vasodilator synthesis during lung development. Early Hum Dev 54:271-294

7. Villamor E, Perez-Vizcaino, Cogolludo AL, Conde-Oviedo J, Zaragoza-Arnaez F, Lopez-Lopez JG, Tamargo J 2000 Relaxant effects of carbon monoxide compared with nitric oxide in pulmonary and systemic vessels of newborn piglets. Pediatr Res 48:546-553

8. Pérez-Vizcaíno F, López-López JG, Santiago R, Cogolludo A, Zaragozá-Arnáez F, Moreno L, Alonso MJ, Salaíces M, Tamargo J 2002 Postnatal maturation in nitric oxide-induced pulmonary artery relaxation involving cyclooxygenase- 1 activity. Am J Physiol Lung Cell Mol Physiol 283:L839-L848

9. Abman SH 1999 Abnormal vasoreactivity in the pathophysiology of persistent pulmonary hypertension of the newborn. Pediatr Rev 20:e103-e109

10. Lucas KA, Pitari GM, Kazerounian S, Ruiz-Stewart I, Park J, Schulz S, Chepenik KP, Waldman SA 2000 Guanylyl cyclases and signaling by cyclic GMP. Pharmacol Rev 52:375-414

11. Francis SH, Turko IV, Corbin JD 2001 Cyclic nucleotide phosphodiesterases: relating structure and function. Prog Nucleic Acid Res Mol Biol 65:1-52

12. Rybalkin SD, Yan C, Bornfeldt KE, Beavo JA 2003 Cyclic GMP phosphodiesterases and regulation of smooth muscle function. Circ Res 93:280-291 
13. Giordano D, De Stefano ME, Citro G, Modica A, Giorgi M 2001 Expression of cGMP-binding cGMP-specific phosphodiesterase (PDE5) in mouse tissues and cell lines using an antibody against the enzyme amino-terminal domain. Biochim Biophys Acta 1539:16-27

14. MacLean MR, Johnston ED, McCulloch KM, Pooley L, Houslay MD, Sweeney G 1997 Phosphodiesterase isoforms in the pulmonary arterial circulation of the rat: changes in pulmonary hypertension. J Pharmacol Exp Ther 283:619-624

15. Black SM, Sanchez LS, Mata-Greenwood E, Bekker JM, Steinhorn RH, Fineman JR 2001 sGC and PDE5 are elevated in lambs with increased pulmonary blood flow and pulmonary hypertension. Am J Physiol Lung Cell Mol Physiol 281:L1051-L1057

16. Hanson KA, Ziegler JW, Rybalkin SD, Miller JW, Abman SH, Clarke WR 1998 Chronic pulmonary hypertension increases fetal lung cGMP phosphodiesterase activity. Am J Physiol 275:L931-L941

17. Ghofrani HA, Wiedemann R, Rose F, Schermuly RT, Olschewski H, Weissmann N, Gunther A, Walmrath D, Seeger W, Grimminger F 2002 Sildenafil for treatment of lung fibrosis and pulmonary hypertension: a randomised controlled trial. Lancet 360:895-900

18. Zhao L, Mason NA, Morrell NW, Kojonazarov B, Sadykov A, Maripov A, Mirrakhimov MM, Aldashev A, Wilkins MR 2001 Sildenafil inhibits hypoxia-induced pulmonary hypertension. Circulation 104:424-428

19. Michelakis E, Tymchak W, Lien D, Webster L, Hashimoto K, Archer S 2002 Ora sildenafil is an effective and specific pulmonary vasodilator in patients with pulmonary arterial hypertension: comparison with inhaled nitric oxide. Circulation 105:2398-2403

20. Shekerdemian LS, Ravn HB, Penny DJ 2002 Intravenous sildenafil lowers pulmonary vascular resistance in a model of neonatal pulmonary hypertension. Am J Respir Crit Care Med 165:1098-1102

21. Oliver J, Webb DJ 2002 Sildenafil for "blue babies". Such unlicensed drug use might be justified as a last resort. BMJ 325:1174

22. Hanson KA, Burns F, Rybalkin SD, Miller JW, Beavo J, Clarke WR 1998 Developmental changes in lung cGMP phosphodiesterase-5 activity, protein, and message. Am J Respir Crit Care Med 158:279-288

23. Black SM, Johengen MJ, Soifer SJ 1998 Coordinated regulation of genes of the nitric oxide and endothelin pathways during the development of pulmonary hypertension in fetal lambs. Pediatr Res 44:821-830

24. Sanchez LS, de la Monte SM, Filippov G, Jones RC, Zapol WM, Bloch KD 1998 Cyclic-GMP-binding, cyclic-GMP-specific phosphodiesterase (PDE5) gene expression is regulated during rat pulmonary development. Pediatr Res 43:163-168

25. Bina S, Hart JL, Sei Y, Muldoon SM 1998 Factors contributing to differences in the regulation of cGMP in isolated porcine pulmonary vessels. Eur J Pharmacol 351:253-260
26. Cortijo J, Naline E, Ortiz JL, Berto L, Girard V, Malbezin M, Advenier C, Morcillo EJ 1998 Effects of fenspiride on human bronchial cyclic nucleotide phosphodiesterase isoenzymes: functional and biochemical study. Eur J Pharmacol 341:79-86

27. Rousseau E, Gagnon J, Lugnier C 1994 Characterization of cyclic nucleotide phosphodiesterase isozymes in bovine airway epithelium. Mol Cell Biochem 140:171-175

28. Rousseau E, Dostie J, Taoudi-Benchekroun M, Cadieux A, Beaudry C, Lugnier C 1995 Specific cyclic nucleotide phosphodiesterase inhibitors differently modulate contractile kinetics in airway smooth muscle. Can J Physiol Pharmacol 73:1784-1794

29. Lopez-Lopez JG, Perez-Vizcaíno F, Cogolludo AL, Ibarra M, Zaragoza-Arnáez F, Tamargo J 2001 Nitric oxide- and nitric oxide donors-induced relaxation and its modulation by oxidative stress in piglet pulmonary arteries. Br J Pharmacol 133:615-624

30. Keravis TM, Wells JN, Hardman JG 1980 Cyclic nucleotide phosphodiesterase activities from pig coronary arteries. Lack of interconvertibility of major forms. Biochim Biophys Acta 613:116-129

31. Pauvert O, Salvail D, Rousseau E, Lugnier C, Marthan E, Savineau JP 2002 Characterisation of cyclic nucleotide phosphodiesterase isoforms in the media layer of the main pulmonary artery. Biochem Pharmacol 63:1763-1772

32. Pauvert O, Lugnier C, Keravis T, Marthan R, Rousseau E, Savineau JP 2003 Effect of sildenafil on cyclic nucleotide phosphodiesterase activity, vascular tone and calcium signaling in rat pulmonary artery. Br J Pharmacol 139:513-522

33. Loughney K, Hill TR, Florio VA, Uher L, Rosman GJ, Wolda SL, Jones BA, Howard ML, McAllister-Lucas LM, Sonnenburg WK, Francis SH, Corbin JD, Beavo JA, Ferguson K 1998 Isolation and characterization of cDNAs encoding PDE5A, a human cGMP-binding, cGMP-specific 3',5'-cyclic nucleotide phosphodiesterase. Gene 216:139-147

34. Wu SN, Li HF, Chiang HT 2001 Vinpocetine-induced stimulation of calcium-activated potassium currents in rat pituitary GH3 cells. Biochem Pharmacol 61:877-892

35. Yan C, Zhao AZ, Bentley JK, Beavo JA 1996 The calmodulin-dependent phosphodiesterase gene PDE1C encodes several functionally different splice variants in a tissue-specific manner. J Biol Chem 271:25699-25706

36. Rybalkin SD, Rybalkina IG, Feil R, Hofmann F, Beavo JA 2002 Regulation of cGMP-specific phosphodiesterase (PDE5) phosphorylation in smooth muscle cells. J Biol Chem 277:3310-3317

37. Finer NN, Barrington KJ 2001 Nitric oxide for respiratory failure in infants born at or near term. Cochrane Database Syst Rev 4:CD000399

38. Reffelmann T, Kloner RA 2003 Therapeutic potential of phosphodiesterase 5 inhibition for cardiovascular disease. Circulation 108:239-244

39. Shekerdemian LS, Ravn HB, Penny DJ 2004 Interaction between inhaled nitric oxide and intravenous sildenafil in a porcine model of meconium aspiration syndrome. Pediatr Res 55:413-418 